\title{
LA ASAMBLEA INTERNACIONAL 2003-2004 DE LOS TESTIGOS DE JEHOVÁ ¿UNA NUEVa OpORTUNIDAD EN MÉxico?
}

\author{
Antonio Higuera Bonfil
}

\section{Introducción}

$\mathrm{H}$ ace casi un siglo que los testigos de Jehová iniciaron su labor proselitista en México. La génesis de este quehacer sólo implicó a unos cuantos predicadores, quienes se dieron a la tarea de promover su religión en un país incuestionablemente católico, en el que además un abanico de confesiones religiosas originales e importadas había encontrado acomodo.

Si prestamos oídos a la leyenda, habría que decir que fueron unos cuantos individuos los que se impusieron — en los albores del siglo xx — la tarea de difundir en nuestro país las ideas de los Estudiantes Internacionales de la Biblia, primer nombre que adoptaron los testigos de Jehová. Al parecer, dichos prolegómenos pasaron varios años en Estados Unidos, lugar en el que un cuarto de siglo antes Charles T. Russell iniciara una organización religiosa que en sólo una centuria observaría un crecimiento sin precedente.

Hoy, la Sociedad torre del vigía reivindica la existencia de 6429351 testigos de Jehová en el mundo, de los cuales, 988236 radican en Estados Unidos, 576 069 en Brasil y 555653 se encuentran en México. Hay que decir que un grupo religioso como éste, que reclama su presencia en 235 países (La torre del Vigía, 2004: 31-38) alcanza ya determinados niveles de organiza- ción y ha evolucionado de tal forma que diversos autores señalan que dejaron de ser una secta, en el estricto sentido sociológico del concepto, para convertirse en una secta establecida. ${ }^{1}$

Una expresión de la complejidad alcanzada por La torre del vigía es la realización de asambleas que reúnen un número determinado de congregaciones; en estas ocasiones se llevan adelante actividades específicas que buscan reforzar el compromiso de bautizados y adherentes, son además el escenario de la incorporación formal de nuevos prosélitos.

La torre del vigía concibe estas reuniones de la siguiente forma:

Las asambleas de distrito, circuito e internacionales [...] han provisto el marco para la revelación de más luz sobre la verdad y la presentación de nuevas publicaciones para el estudio personal y de congregación y para ser usadas en el ministerio del campo, así como para los bautismos en masa.

[...] Nuestro envolvimiento en las reuniones locales de congregación, así como en las reuniones mayores, del tipo de asambleas de circuito y distrito [...] nos fortalece para hacer la voluntad de Jehová y nos protege contra las influencias mundanas exteriores que atacan nuestra fe cristiana con el propósito de socavarla (Franz, 1989: 79-80) 
La asambleas de distrito y circuito se realizan una y dos veces al año respectivamente. En la actualidad, las internacionales se verifican cada lustro.

En el caso de las asambleas internacionales, encontramos su antecedente más lejano en una reunión celebrada en Cleveland, Ohio, en 1946, de la que se dice congregó a más de 80000 asistentes de 33 países. Cinco años después se verificó la primera gran asamblea fuera de Estados Unidos, el escenario fue el Estadio Wembley de Londres, donde estuvieron presentes testigos de 40 países. Con este impulso, durante la década de 1950 se realizaron reuniones de este tipo en Alemania e Italia (Henschel, 1993: 270-276).

Fue en 1963 cuando se celebró en forma lo que hoy se conoce como una asamblea internacional; a partir de ese momento la meta fue celebrar estas reuniones con el mismo programa en cada uno de los países seleccionados. Ésta, de hace 41 años, arrancó en Milwuakee siguiendo una ruta que incluyó primero a Nueva York en Estados Unidos para luego pasar a algunas ciudades de Europa, Medio Oriente, la India, Birmania, Tailandia, Honk
Kong, Singapur, Filipinas, Indonesia, Australia, Taiwán, Japón, Nueva Zelanda, Fiji,la República de Coreay Hawai, para finalizar donde empezó, en Estados Unidos.

En 1966 se verificó la primera asamblea internacional en América Latina, mientras que el continente africano fue escenario de este tipo de eventos a partir de 1970 (Henschel, 1993: 276-277) Como puede apreciarse con estos primeros datos, se trata de una tradición que pronto tendrá medio siglo de existencia y parece que sus metas esenciales se han logrado: demostrar públicamente el avance de esta confesión religiosa y tratar de alcanzar a la mayor población posible.

En este texto se presentará una hipótesis de trabajo, que apunta hacia la conclusión de un ciclo de crecimiento de los testigos de Jehová en México, lo que daría paso a una nueva etapa, que privilegiaría la continuidad de su obra en el mediano plazo. La asamblea internacional denominada Demos gloria a Dios, celebrada en México del 5 al 9 de noviembre de 2003, es un buen ejemplo del proceso que se postula está experimentando la organización central de los testigos de Jehová en los últimos años.

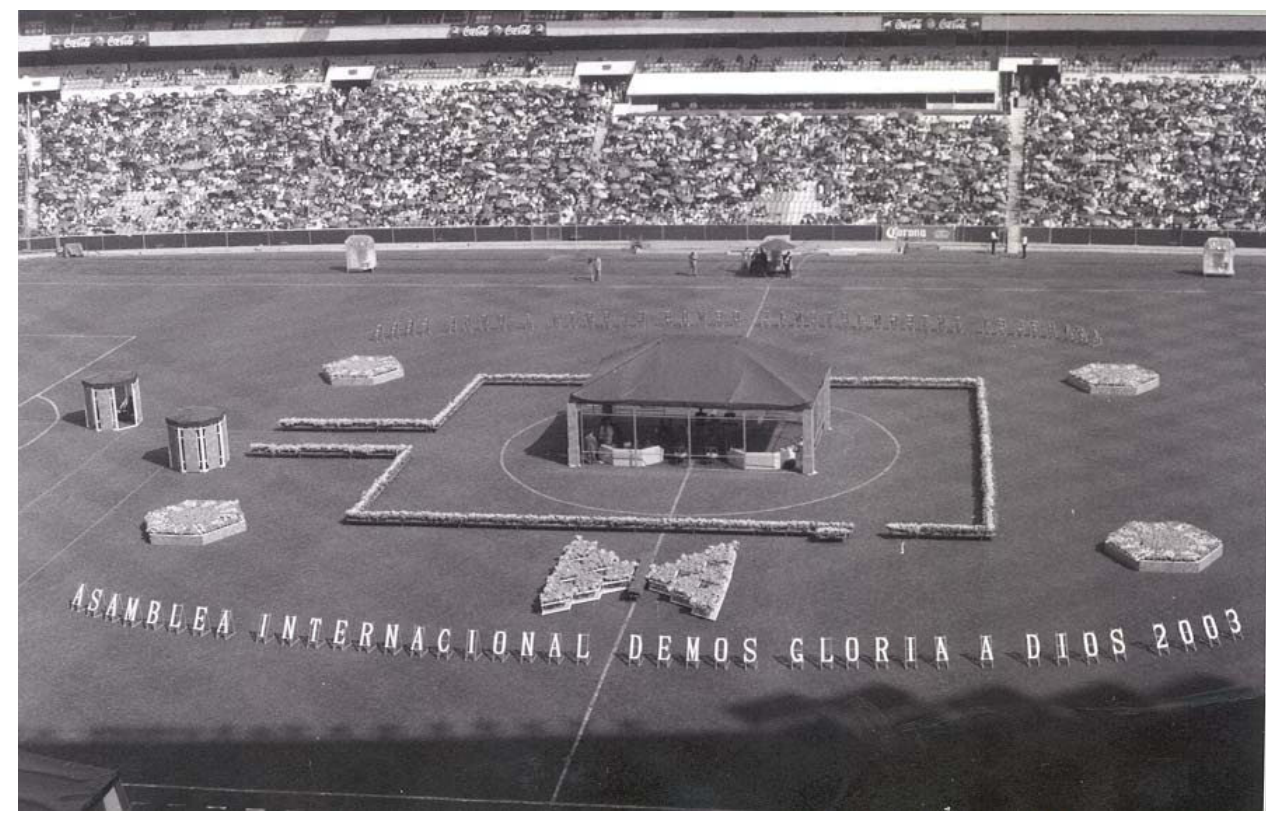


Así pues, nuestra atención se centrará en el programa de dicha asamblea, sabiendo que hay muchos otros elementos que apuntan en la misma dirección, pero que no sería posible abordar en esta ocasión. ${ }^{2}$ No puede dejar de mencionarse el hecho de que la Sociedad torre del vigía organizó el año pasado 30 asambleas, que tuvieron lugar en 29 ciudades de 14 países.

\section{La asamblea internacional}

El primer punto de referencia es el hecho que desde hacía 25 años México no era escenario de una asamblea internacional de los testigos de Jehová. ${ }^{3}$ De acuerdo con la literatura producida por La torre del vigía, en nuestro país se celebró este tipo de encuentros en 1945 y 1966 ,

(...) La Asamblea Internacional Voluntad Divina, que se celebró en 1958 en Nueva York, fue sobresaliente al respecto. Estuvieron presentes personas de 123 países, incluidas 503 de México, (...) Otra asamblea conmovedora fue la que se celebró en la Ciudad de México en 1966, a la que asistieron centenares de representantes de una docena de países que viajaron a México para estar con sus hermanos en la reunión más grande organizada por La Torre del Vigía de México hasta esa fecha. En aquella ocasión, la asistencia fue treinta veces mayor que la de la asamblea de 1945.

(Henschel, 1995: 217)

Con este antecedente, parece lógico que una ocasión como ésta no sería de menor importancia para los testigos mexicanos. Participaron en la organización y desarrollo de la asamblea alrededor de 36000 personas. Hay que anotar desde ahora que la asamblea tuvo tres escenarios en México: el Estadio Azteca, en la Ciudad de México, el Cuauhtémoc en Puebla (6-9 de noviembre) y el de beisbol Monterrey en esa ciudad regiomontana (69 de noviembre). ${ }^{4}$
A la asamblea asistieron - repartidos en los tres escenarios- 5200 delegados internacionales de 16 países, incluidos 3 de África. ${ }^{5}$ La recopilación de datos etnográficos se realizó en el Estadio Azteca los cuarto días de asamblea y se utilizó tanto la observación directa como la participante. Se tiene registrado $100 \%$ del programa del evento en cintas de audio y se recibió algún material impreso, además de efectuar entrevistas formales e informales.

Los datos sobre la magnitud de este evento hace evidente la necesidad de una maquinaria bien aceitada para coordinar la reunión, que involucra no sólo a muchas personas, sino que implica arreglos de invitación a delegados internacionales, alojamiento, seguridad, servicios básicos en el estadio, primeros auxilios, objetos perdidos y guardarropa, sonido, limpieza, acomodo de asistentes, etcétera.

Por ello es la sede mundial de los testigos la que define en qué países tendrán lugar estas asambleas internacionales; parece un requisito insalvable el que las sedes tengan una feligresía lo suficientemente grande y comprometida como para asumir las responsabilidades que conlleva este evento. No sólo se buscan grandes ciudades con suficientes congregaciones, sino que se consideran elementos tales como la actitud del gobierno local hacia la labor de los testigos y su respuesta ante la magnitud y significación del evento, la facilidad en las comunicaciones (usualmente se requiere de ciudades con aeropuertos internacionales) y el tener un inmueble con un aforo importante, entre otros.

Tras estas y otras consideraciones, el cuerpo gobernante de los testigos de Jehová invita a las sucursales nacionales como anfitrionas. Establecido este compromiso, el trabajo de organización y coordinación da sus primeros pasos; como el programa de la asamblea internacional coincide en un alto porcentaje con el de una asamblea de distrito ordinaria, ${ }^{6}$ son los superintendentes de circuito los encargados de proponer a la sucursal nacional los candidatos que fungirían como coordinadores de los trabajos. 
Se afina un organigrama en el que se asientan las necesidades concretas de cada área y se estipula el número aproximado de colaboradores que se requeriría para su satisfacción. El siguiente paso es designar a los encargados de aproximadamente veinte departamentos que atenderán aspectos concretos del evento. De esta forma, alrededor de cien testigos con privilegios fueron designados para organizar y desarrollar tareas concretas: plataforma y sonido, escenario, limpieza, mantenimiento, acomodadores o alojamiento son algunos de esos departamentos coordinados por el de administración, que hace las veces de comité de asamblea.?

Tras un estimado de la encomienda, cada superintendente de departamento determina cuántos auxiliares requiere y atendiendo las recomendaciones de los superintendentes de circuito, que conocen con cierto detalle a los miembros de unas veinte congregaciones, selecciona a personas que tienen determinados perfiles útiles para las labores asignadas.

De esta forma, seis meses antes de la asamblea se iniciaron los trabajos previos y se dividió el Estadio Azteca en secciones (a su vez se subdividen en otras más pequeñas) que fueron asignadas a los auxiliares de cada departamento. ${ }^{8}$ Con alguna antelación se revisó y dio mantenimiento a la red hidráulica del coloso de Santa Úrsula, se dejaron a punto los servicios sanitarios y se verificó que la red eléctrica del estadio reuniera las condiciones necesarias.

Una semana antes del inicio de la asamblea, los departamentos efectuaron tareas concretas para asegurar condiciones básicas de funcionamiento; se entrenaron a los acomodadores en las actividades específicas de dar acceso y orientación a los asistentes, se montaron tiendas de campaña para ofrecer servicios como el de primeros auxilios, alojamiento, objetos perdidos y guardarropa. Los preparativos se concluyeron y aun se tenía lista una serie de actividades ofrecidas por la sucursal nacional a los delegados internacionales, que iban desde la recepción en el aeropuerto internacional de la Ciudad de México y la atención global de las delegaciones, hasta las reuniones sociales que incluían tanto convivencia con betelitas, giras por la misma sucursal, así como espectáculos que muestran elementos del folclore mexicano.

Un aspecto que debe tratarse por separado es el referente a las autorizaciones del gobierno local para llevar adelante la asamblea internacional. De acuerdo con lo señalado por el departamento de administración, para el caso que nos ocupa la sucursal nacional de los testigos de Jehová presentó una solicitud ante la Secretaría de Gobernación y tramitó los permisos necesarios ante la delegación política correspondiente. ${ }^{9}$

No dejamos fuera de esta reseña el hecho de que hay cuestiones que no pueden ser resueltas directamente por los organizadores de la asamblea. Por ello hay que decir que se contratan servicios específicos; el inmueble que hace las veces de salón del reino es rentado por la sucursal nacional para eventos semejantes al que nos interesa, desde 1999 y ésta fue la cuarta ocasión en que se celebró una asamblea en el Estadio Azteca. El agua para los servicios básicos no puede quedar al garete, por ello se cuenta con pipas de agua que abastecen los sanitarios y se asegura la limpieza del recinto.

Aunque las recomendaciones de la sucursal de resolver individual o familiarmente los asuntos de bastimento son generalmente atendidas por los asistentes, los cuatro días de asamblea se observó que los puestos de comida y bebidas no embriagantes del interior del Azteca funcionaron normalmente, pero es cierto que lo hicieron en un horario restringido.

Si bien como ya se mencionó, el programa de esta asamblea internacional es muy semejante al de una asamblea de distrito, es de resaltarse que la participación de los delegados internacionales se manifiesta no sólo en el informe de las actividades de sus respectivos países, sino en un intenso proceso de convivencia e intercambio de experiencias de vida, tanto dentro como fuera del temporal salón del reino se aprovecharon las oportunidades para conocer de cerca qué panorama hay en la labor 
religiosa que desempeñan en cada nación y se enteran de cómo marchan las cosas en las diferentes latitudes.

A pesar de ser una imagen sencilla la que se ha presentado hasta aquí, dejaremos esta reseña confirmando que los elementos vertidos son — grosso modo — un botón de muestra del nivel de avance logrado por La torre del vigía en México. Antes de pasar a otro apartado de este trabajo, permítaseme considerar algunos datos que muestran aquella evolución en nuestro país.

Tabla 1

Testigos de Jehová en México y el mundo. Porcentaje de crecimiento anual en los dos ámbitos

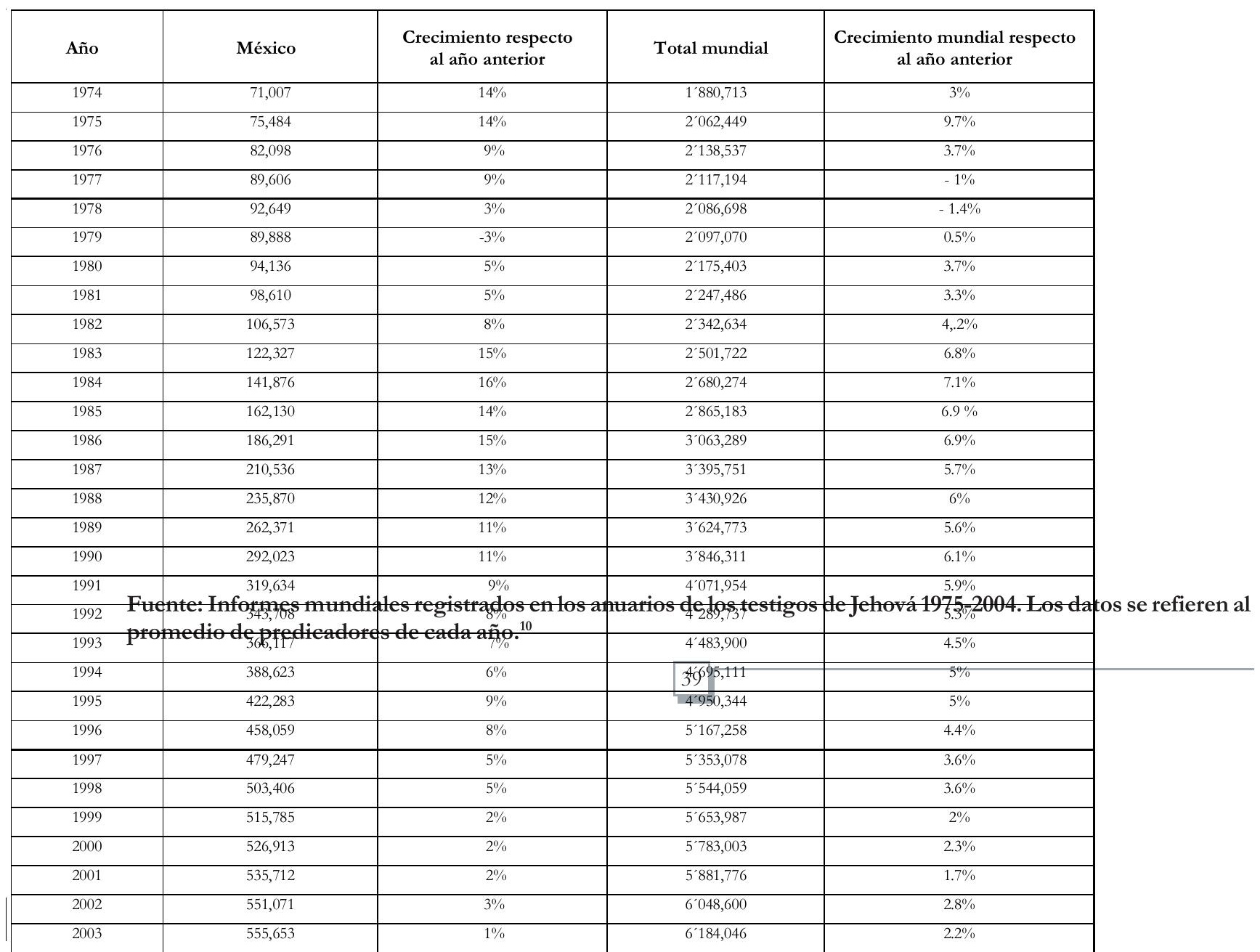


La tabla 1 contiene los datos de la conversión anual en México durante las últimas tres décadas. De esta serie de treinta años, sólo uno (1979) registra un decremento en la cantidad de predicadores, mientras que en los veintinueve restantes se observa un incremento de intensidad variable.

Llama nuestra atención la variación en el ritmo de crecimiento. Si bien en más de 95\% del periodo se experimentó incremento en el número de los testigos mexicanos, al inicio de la primera década y durante la mayor parte de la segunda, se constata un crecimiento mayor a $10 \%$ respecto al año anterior. Parece claro que asistimos a uno de los más importantes periodos de crecimiento de La torre del vigía en México. Si bien es cierto que de 1931 a 1974 — cuando se pasó de 82 a 70000 conversos- puede considerarse como el lapso de establecimiento definitivo de los testigos de Jehová en el país, es la década de 1980 la que observa un crecimiento sin precedente, pasando de algo más de ciento veinte mil testigos a prácticamente trescientos mil.

Sin embargo, de 1976 a 1982 este porcentaje baja a un dígito y en este periodo está aquel año en que se registró el único decremento de la serie considerada. Creemos que un importante elemento para explicar esta variación lo constituye el papel asignado al año 1975. De acuerdo con los cálculos de La torre del vigía, sería entonces que concluirían los seis mil años de vida terrena y sobrevendría el fin del mundo - no un fin literal, sino de los términos en que conocemos y funciona actualmente el mundo-(Henschel, 1993).

La expectativa generada pudiera explicar, aunque sea en parte, por qué hasta ese año el crecimiento de los testigos en México fue realmente asombroso y por qué a partir de 1976, cuando el mundo no modificó sus condiciones generales, sobrevino el desencanto y la consecuente reducción en el crecimiento durantelos siguientes siete años.

Finalmente, la misma tabla muestra que de 1991 a 2003 vuelve a reducirse el porcentaje de crecimiento anual a un solo dígito; de hecho en los últimos cinco años lo que ha ocurrido es un crecimiento marginal de no más de $3 \%$. Estos datos indican que, como nunca antes, la labor de los testigos en nuestro país se ha deprimido significativamente y se encuentra en una etapa de letargo que, por lo que se dirá en la segunda parte de este trabajo, pudiese tratarse de un periodo de reorganización.

La gráfica 1, que considera los últimos treinta años en México, permite ver que se pasó de setenta y un mil testigos a más de quinientos cincuenta mil, lo que representa un crecimiento de casi $800 \%$. Como puede apreciarse, la curva de crecimiento habla por sí misma y habría que estar pendientes de lo que ocurra los siguientes años, de ahí la pertinencia de preguntarse si se consolidará la tendencia del crecimiento marginal o, por el contrario, se retomará el ritmo acelerado de la década de $1980 \ldots$

\section{Una comparación que ilustra}

Como se señaló al inicio de esta presentación, nuestra hipótesis de trabajo apunta hacia la conclusión de una etapa de crecimiento de los testigos en México y el advenimiento de un momento de reorganización. Aunque hay circunstancias cualitativas que marcan una diferencia en muchos terrenos, pero creyendo que resultaría ilustrativo hacer un breve ejercicio comparativo, se pone a su consideración los datos del crecimiento de esta confesión en Estados Unidos para el periodo 1974-2003.

Hay que señalar que el surgimiento en ese país de la Sociedad Watch Tower durante la recta final del siglo XIX, el establecimiento de las oficinas centrales en esa nación, la labor de Charles T. Russell — su fundador y primer presidente-, así como de los siguientes cinco presidentes corporativos en el terreno del proselitismo, obtuvo sus primeros resultados en Estados Unidos. Fue desde este punto donde se inició y se sigue coordinando la prédica global de los testigos de Jehová, por lo que 


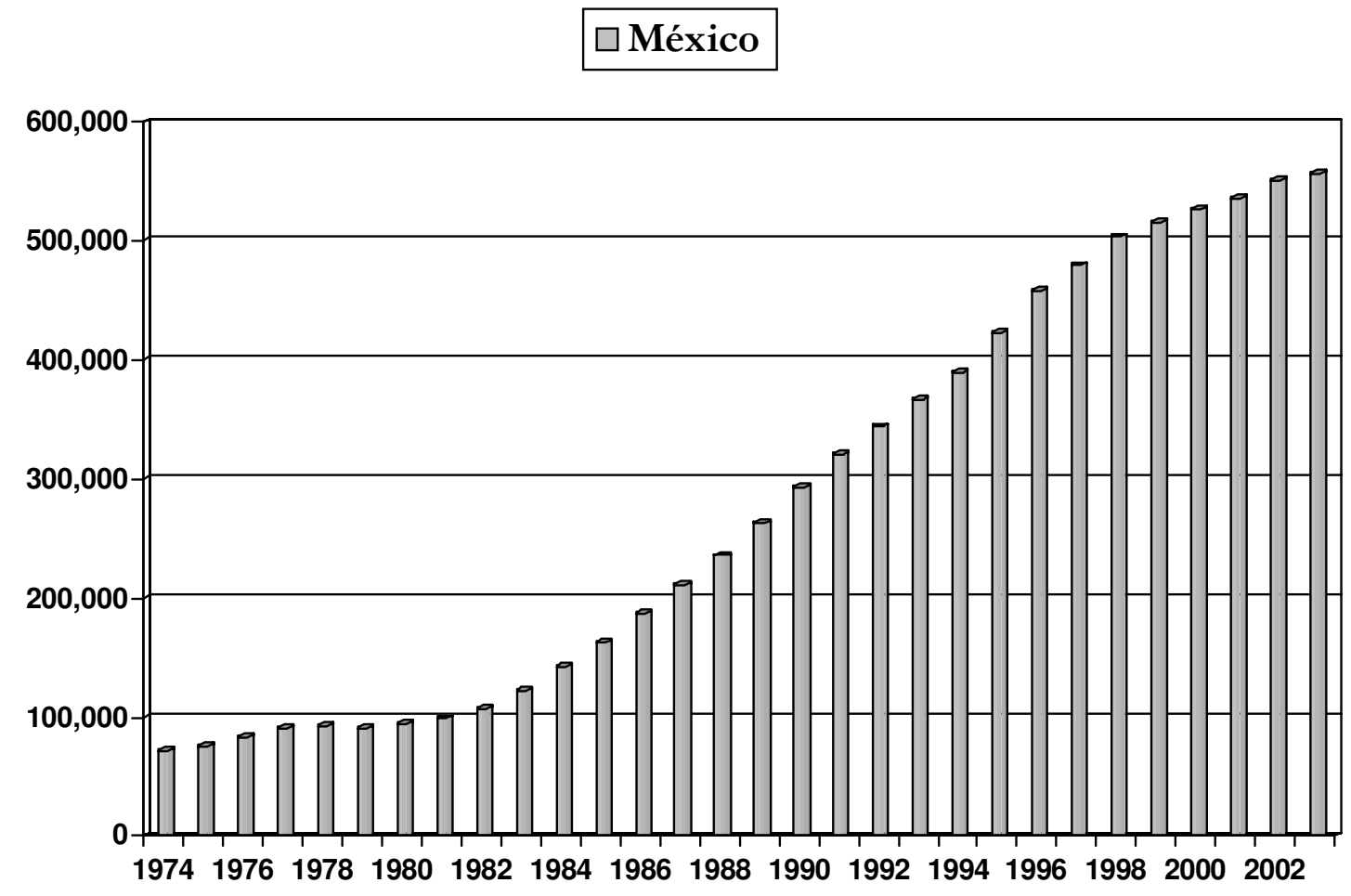

observar su crecimiento y cotejarlo con lo ocurrido en México permitirá tanto apreciar los procesos particulares de cada país, como contar con más elementos para comprender el momento que se vive en nuestro país.

La tabla 2 muestra el crecimiento anual de los testigos estadunidenses durante las últimas tres décadas. Una primera semejanza con el caso mexicano es que cerca de 1975 — cuando las condiciones del mundo no cambiaron como se esperaba - se observa un decremento en el número de testigos de ese país; el resto de la serie muestra la tendencia que caracteriza al periodo: un crecimiento marginal (de menos de 5\%) en veintidós años, mientras que hay cinco años donde el crecimiento es ligeramente mayor, pero nunca supera el 6 por ciento.

Resalta el hecho de que sólo 1974, año inicial de la serie, muestra un crecimiento superior a 10\%, y deja abierta la pregunta sobre lo que ocurrió en el periodo inmediato anterior. ${ }^{11}$

Estos primeros indicadores pueden complementarse observando el grado de crecimiento de los testigos en Estados Unidos. La gráfica 2 muestra la curva de incremento para el periodo de treinta años y señala que de algo más de quinientos mil testigos se pasó a casi un millón para el año 2003. Si comparamos esta cifra con lo ocurrido en México confirmaremos que mientras Estados Unidos duplicó su número mediante un crecimiento marginal en veintidós años, en México se multiplicó ocho veces la cantidad de testigos y habiendo llegado al medio millón, se entró en la etapa de crecimiento marginal, que sigue vigente.

No dejamos de entender que hay una diferencia significativa entre los datos absolutos y los relativos. Sin 
T E

Tabla 2

Testigos de Jehová en Estados Unidos y el mundo.

Porcentaje de crecimiento anual en los dos ámbitos

\begin{tabular}{|c|c|c|c|c|}
\hline Año & Estados Unidos & $\begin{array}{l}\text { Crecimiento respecto } \\
\text { al año anterior }\end{array}$ & Total mundial & $\begin{array}{l}\text { Crecimiento mundial } \\
\text { respecto al año anterior }\end{array}$ \\
\hline 1974 & 506367 & $15 \%$ & 1880713 & $3 \%$ \\
\hline 1975 & 534765 & $6 \%$ & 2062449 & $9.7 \%$ \\
\hline 1976 & 544644 & $2 \%$ & 2138537 & $3.7 \%$ \\
\hline 1977 & 530374 & $-3 \%$ & 2117194 & $-1 \%$ \\
\hline 1978 & 513673 & $-3 \%$ & 2086698 & $-1.4 \%$ \\
\hline 1979 & 521370 & $1 \%$ & 2097070 & $0.5 \%$ \\
\hline 1980 & 543457 & $4 \%$ & 2175403 & $3.7 \%$ \\
\hline 1981 & 563452 & $4 \%$ & 2247486 & $3.3 \%$ \\
\hline 1982 & 581934 & $3 \%$ & 2342634 & $4, .2 \%$ \\
\hline 1983 & 616058 & $6 \%$ & 2501722 & $6.8 \%$ \\
\hline 1984 & 648704 & $5 \%$ & 2680274 & $7.1 \%$ \\
\hline 1985 & 678510 & $5 \%$ & 2865183 & $6.9 \%$ \\
\hline 1986 & 710344 & $5 \%$ & 3063289 & $6.9 \%$ \\
\hline 1987 & 734378 & $3 \%$ & 3395751 & $5.7 \%$ \\
\hline 1988 & 762960 & $4 \%$ & 3430926 & $6 \%$ \\
\hline 1989 & 788709 & $3 \%$ & 3624773 & $5.6 \%$ \\
\hline 1990 & 816417 & $4 \%$ & 3846311 & $6.1 \%$ \\
\hline 1991 & 846028 & $4 \%$ & 4071954 & $5.9 \%$ \\
\hline 1992 & 866362 & $2 \%$ & 4289737 & $5.3 \%$ \\
\hline 1993 & 878841 & $1 \%$ & 4483900 & $4.5 \%$ \\
\hline 1994 & 889570 & $1 \%$ & 4695111 & $5 \%$ \\
\hline 1995 & 912002 & $3 \%$ & 4950344 & $5 \%$ \\
\hline 1996 & 921123 & $1 \%$ & 5167258 & $4.4 \%$ \\
\hline 1997 & 929471 & $1 \%$ & 5353078 & $3.6 \%$ \\
\hline 1998 & 944218 & $2 \%$ & 5544059 & $3.6 \%$ \\
\hline 1999 & 940650 & $-0.3 \%$ & 5653987 & $2 \%$ \\
\hline 2000 & 945000 & $0.4 \%$ & 5783003 & $2.3 \%$ \\
\hline 2001 & 945689 & $0.7 \%$ & 5881776 & $1.7 \%$ \\
\hline 2002 & 972754 & $3 \%$ & 6048600 & $2.8 \%$ \\
\hline 2003 & 988236 & $2 \%$ & 6184046 & $2.2 \%$ \\
\hline
\end{tabular}

Fuente: Informes mundiales registrados en los anuarios de los testigos de Jehová 1975-2004. Los datos se refieren al promedio de predicadores de cada año. 
T E

embargo, guardadas las proporciones del caso, parece adecuado considerar que hay una interesante semejanza en cuanto al proceso vivido en ambos países. Tal condición no coincide en el plano temporal, sino en el modelo, cuya tendencia de comportamiento se repite en los dos escenarios: una intensa expansión que consolida importantes números de conversión, luego una fase de receso que supone una reorganización para ganar nuevos adeptos.

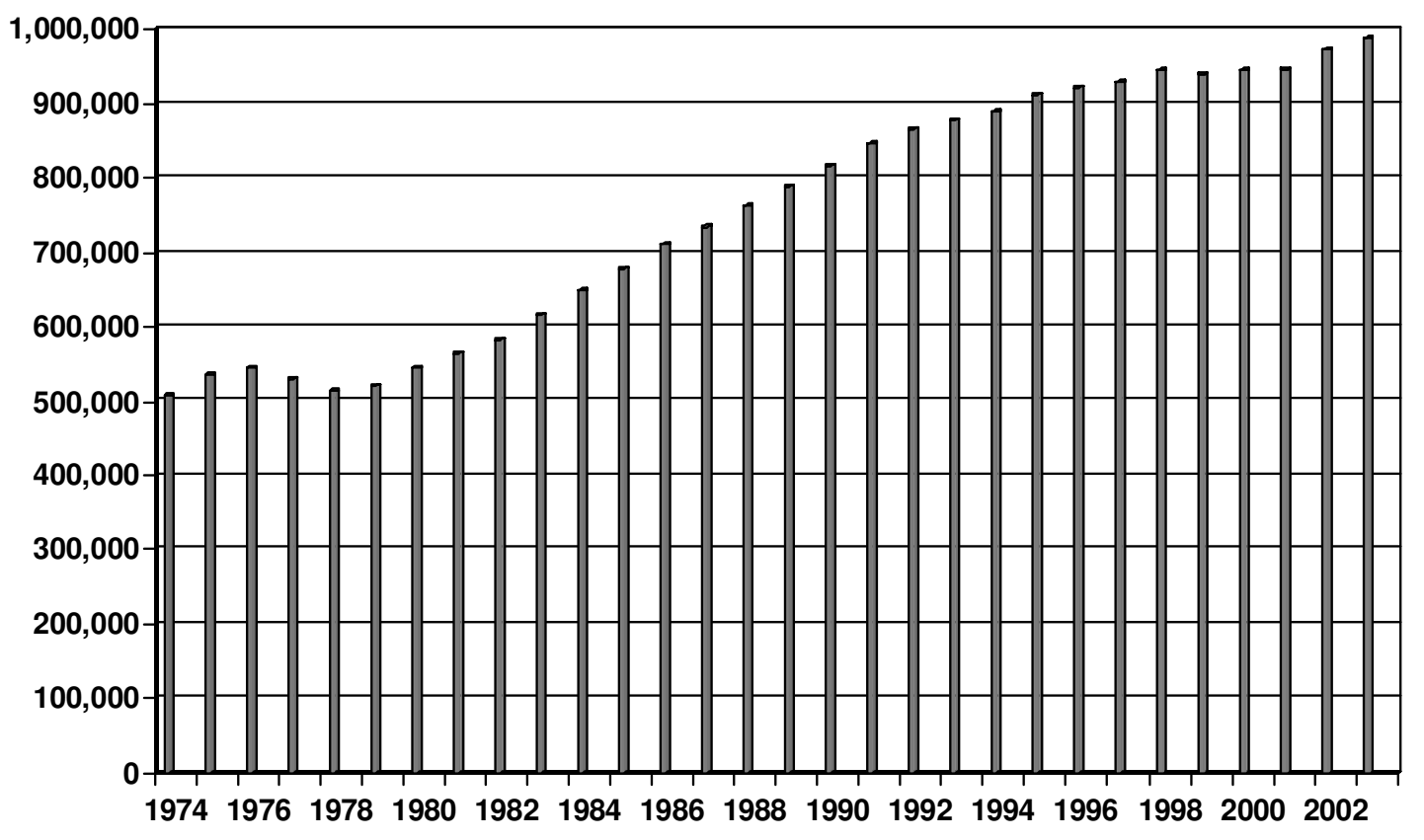

$\square$ Estados Unidos

\section{La nueva estrategia}

Para concluir este texto pongo a su consideración algunos elementos que integran la etapa de reorganización que se experimenta en la sucursal mexicana. Soy de la opinión de que se pueden identificar acciones y directrices que buscan incrementar el ritmo proselitista de los testigos.

Volviendo a la asamblea internacional de 2003, se constató la existencia de determinados cambios, que si bien por sí mismos difícilmente apoyan nuestra hipóte- sis, pueden ser considerados en adición a ciertas actividades cotidianas de La torre del vigía y que en conjunto aportarían una perspectiva diferente.

¿A qué cambios me refiero? En primer lugar habría que decir que el programa de la asamblea (que es internacional y cuyo contenido debe aplicarse lo más posible) incluye diferentes tópicos para fortalecer las relaciones al interior de las familias, poniendo especial énfasis en la vinculación entre padres e hijos y llamando la atención de aquéllos para atender y guiar a los que están bajo su cuidado. 
Resulta de interés observar que se fomenta la lectura diaria de la Biblia en el seno familiar, para ello La torre del vigía produce desde hace varios años ediciones anuales que contienen la programación de lectura durante el año en curso. ${ }^{12}$ Esta práctica cotidiana se ve reforzada en las reuniones de congregación y por otros elementos de estudio cotidiano de los testigos: El ministerio del reino y La atalaya incluyen la consideración de aquel texto.

En el contexto mexicano, donde como se ha visto se experimenta un crecimiento marginal, esta directriz es particularmente importante, toda vez que apuntala la consolidación de la formación de niños nacidos en el seno de familias testigos de Jehová.

Por otro lado, es especialmente interesante haber confirmado que al interior de las prácticas ordinarias de los testigos de Jehová, la edad requerida para realizar determinadas actividades se ha reducido de una manera importante.

No sólo es cada vez más común encontrar preadolescentes e infantes en las visitas de casa en casa, ofreciendo literatura de la Sociedad o asistiendo a reuniones de congregación y asambleas, sino que este grupo de edad se ha incorporado a actividades que suponen un cierto avance en el conocimiento de los valores y prácticas que caracterizan a los testigos; ya es ordinario observar que pequeños de algo más de diez años suban a la plataforma de los salones del reino y participen en actividades tales como dar testimonio, contestar preguntas sobre La atalaya o dirigir estudios bíblicos en la casa de quienes se muestran interesados por el mensaje de $\mathrm{La}$ torre del vigía.

Otra modificación que apunta en la misma dirección es que habiendo reunido los requisitos marcados por la Sociedad torre del vigía, la edad para el bautismo ha disminuido significativamente. Hace unos diez años lo común era la incorporación de jóvenes adolescentes usualmente mayores a los quince años; en contraste, durante la asamblea internacional se comprobó el que se simbolizaran infantes a partir de los nueve años.
Un ejemplo que va más allá de lo meramente simbólico lo encontramos, también, en la asamblea internacional que nos ocupa. Dos pequeñas publicadoras tuvieron una emotiva y sugerente participación en el programa de esta reunión, ambas dieron testimonio de su desempeño como publicadoras y de viva voz mostraron cómo su condición de infantes no es un obstáculo cuando se proponen llevar adelante las actividades sugeridas por la Sociedad torre del vigía.

Los componentes mencionados en los últimos ejemplos tienen un contexto que los galvaniza y consolida su naturaleza. Se trata, en la mayoría de los casos, de pequeños que han nacido y crecen en el seno de familias de testigos de Jehová. Parece evidente que siendo la descendencia de padres que no comparten la misma religión, haya conflictos para que los niños se involucren a este grado.

En cambio, como se observó en la recopilación de datos etnográficos, cuando un hijo de familia recibe el estímulo de sus dos padres, sigue su ejemplo, y cuenta con el interés manifiesto de la Sociedad torre del vigía para su integración al grupo, entonces la incorporación temprana a la comunidad religiosa es parte del proceso de socialización de los niños; ello culmina en la integración efectiva, en términos no sólo formales sino funcionales, a la confesión religiosa de los progenitores.

Un elemento más que integra el esfuerzo de La torre del vigía por superar el crecimiento marginal de los testigos en México tiene que ver con haber enfocado de manera privilegiada, tal vez como nunca antes, a grupos que habían recibido mínima o nula atención de sus publicadores. Tal vez por ello hoy es común la edición de literatura, que incluye folletos, libros y revistas en diversas lenguas indígenas; también se desarrollan reuniones — estudios biblicos, reuniones de congregación, estudios de La atalaya y aun asambleas de distrito- - para discapacitados.

De esta forma, la actividad cotidiana de diversas congregaciones de testigos de Jehová a lo largo de Méxi- 
co y varias asambleas de distrito que inician en la segunda parte de 2004, se impartirán en lenguas indígenas tales como mazateco, ch'ol, maya yucateco, tseltal, tsotsil, totonaco, zapoteco, purépecha, náhuatl, tojolabal y mixe. De la misma forma, reuniones de este tipo se desarrollarán en lengua de señas mexicana. ${ }^{13}$

Como parece claro, enfocar la labor de proselitismo hacia estos sectores de la sociedad civil —y sumarlo a la población con la que ya se trabaja - podría redituar una opción para superar el crecimiento marginal en que se encuentra la obra en México.

El planteamiento de este trabajo busca encontrar interlocutores para profundizar en el tema abordado. No sólo los científicos sociales, sino quienes siguen la propuesta religiosa de La torre del vigía, pueden ofrecer líneas de discusión y aportes que faciliten la comprensión de diversos aspectos involucrados en una actividad de la complejidad de la que se ha reseñado.

La reflexión sobre el tema propuesto aún tiene aristas por explorar. ¿Qué papel juega —en ese intento por salir del letargo_ la presentación de denuncias ante la Comisión de los derechos humanos cada vez que un niño testigo de Jehová es expulsado de su escuela por no rendir honor a la bandera nacional? ¿Los comités de enlace con hospitales, que desarrollan una labor fundamental en la difusión del uso de la sangre aceptado por los miembros de esta confesión religiosa, colaboran con las familias concretas a mantener sus prácticas culturales y con ello ayudan a mantener núcleos familiares no disgregados en la Organización?

\section{Notas}

${ }^{1}$ Véanse, entre otros, Fortuny, 1994; Garma, 1994, Mann, 1972, Wilson, 1970.

${ }^{2}$ Véanse, entre otros, La familia. Su cuidado y protección. Tratamiento médico para los testigos de Jehová (1995), El secreto de la felicidad familiar (1996), Acerquémonos a Jehová (2002), Aprendamos del gran maestro (2003). Muchos artículos de La Atalaya incluyen el tema, poniendo en relieve la importancia que tienen para La torre del vigía.
Asimismo, los discursos incluidos en asambleas de circuito y distrito de los últimos dos lustros son elementos que deben ser considerados en esta área.

${ }^{3}$ Comunicación personal de José Moreno, betelita de Asuntos Legales de la Sociedad torre del vigía de México, 1 de julio de 2004. ${ }^{4}$ Comunicación personal de José Moreno, betelita de Información Pública de la Sociedad torre del vigía de México, 6 de noviembre de 2003.

${ }^{5}$ De acuerdo con lo expresado por personal del departamento de Información pública de la asamblea, originalmente se esperaba a 15000 delegados.

${ }^{6}$ Entre las diferencias más evidentes se encuentran los reportes que cada país asistente presenta, en un informe especial, el "avance de la Obra" a nivel nacional. La inclusión de discursantes internacionales — de diferentes nacionalidades y residentes en el extranjero — da un matiz particular a esta ocasión, permite conocer la actividad de los misioneros preparados en la Escuela de Gaalad y se difunden experiencias del campo extranjero. Cfr. Programa de la asamblea internacional de los testigos de Jehová 2003-2004 Demos gloria a Dios.

${ }^{7}$ Entrevista formal con Jorge Reues, superintendente del Departamento de Administración de la asamblea internacional Demos gloria a Dios. Estadio Azteca, noviembre 8 de 2003.

${ }^{8}$ Para el caso del Estadio Azteca, el inmueble se dividió en planta baja, plateas, palcos y planta alta. A su vez, éstas se fraccionaron en túneles, accesos y secciones.

${ }^{9}$ Entrevista formal con el superintendente del departamento de administración de la asamblea internacional Demos gloria a Dios. Estadio Azteca, noviembre 8 de 2003. El autor presenció cómo el segundo día de la asamblea se presentó en el departamento de información pública un grupo de personas que se identificaron como empleados de la delegación política de Coyoacán, manifestando estar disponibles a atender lo referente a la seguridad del evento. Al canalizar a este grupo con el departamento de administración, y habiéndoles expresado que tuvieron reuniones de trabajo con el jefe de seguridad pública de esa misma delegación, su actitud se modificó inmediatamente. Felicitaron a los interlocutores por su grado de organización y se retiraron de inmediato.

${ }^{10}$ Las publicaciones consultadas se editaron bajo los siguientes títulos: Libro de textos bíblicos, Libro de actividades y Anuario de los testigos de Jehová. Agradezco a Jesús Herrera Evia, anciano de la congregación Forjadores, de la Ciudad de Chetumal, las facilidades para consultar su biblioteca personal, donde se 
encuentran las ediciones correspondientes a 22 de los 30 años incluidos en esta serie histórica.

${ }^{11}$ Dado los alcances de este trabajo, se deja abierta la pregunta para ser abordada en un trabajo posterior. ${ }^{* * *}$ Los datos consultados a la fecha indican que antes de 1974 el crecimiento en Estados Unidos mantenía cierto ritmo, dándose una clara diferencia con lo observado en el periodo considerado en este trabajo.

${ }^{12}$ Cfr. los folletos Examinando las Escrituras diariamente, correspondientes a años específicos.

${ }^{13}$ Comunicado Asamblea de distrito de los testigos de Jehová "Celosos proclamadores del Reino, mecanuscrito recibido durante la asamblea internacional.

\section{Bibliografía}

CD ROM Watchtower Libary 1995, español.

Fortuny Loret de Mola, Patricia,1994, “Con ellos aprendí a leer: una creencia más intelectual que mística”, en Boletín de la EUCADY, vol. 19, núm 114, Mérida, pp. 41-62.

Franz, F. W., 198?, Organizados para efectuar nuestro ministerio, Grupo Editorial Ultramar, México.

—, 1990, Anuario 1990 de los testigos de Jehová. Con el informe del año de servicio de 1989, Grupo Editorial Ultramarino, México.

—, 1991, Anuario 1991 de los testigos de Jehová. Con el informe del año de servicio de 1990, Grupo Editorial Ultramarino, México.

—, 1991, Anuario 1992 de los testigos de Jehová. Con el informe del año de servicio de, Grupo Editorial Ultramarino, México.

—, 1993, Anuario 1993 de los testigos de Jehová. Con el informe del año de servicio de 1992, Grupo Editorial Ultramarino, México.

Garma Navarro, Carlos, 1994, "El problema de los testigos de Jehová en las escuela mexicanas”, en: Nueva Antropología, Núm. 45, México, El Colegio de México/uam-Iztapalapa, pp. 21-30. Henschel, Milton G., 1993, Los testigos de Jehová. Proclamadores del reino de Dios, Watch Tower Bible and Tract Society of Pennsylvania, México.

—, 1994, Anuario 1994 de los testigos de Jehová. Con el informe del año de servicio de 1993, Grupo Editorial Ultramarino, México.

—, 1995, Anuario 1995 de los testigos de Jehová. Con el informe del año de servicio de 1994, La torre del vigía, México.

—, 1996, Anuario 1996 de los testigos de Jehová. Con el informe del año de servicio de 1995, La torre del vigía, México.

—, 1997, Anuario 1997 de los testigos de Jehová. Con el informe del año de servicio de 1996, La torre del vigía, México.
—, 1998, Anuario 1998 de los testigos de Jehová. Con el informe del año de servicio de 1997, La torre del vigía, México.

—, 1999, Anuario 1999 de los testigos de Jehová. Con el informe del año de servicio de 1998, La torre del vigía, México.

—, 2000, Anuario 2000 de los testigos de Jehová. Con el informe del año de servicio de 1999, La torre del vigía, México.

—, 2001, Anuario 2001 de los testigos de Jehová. Con el informe del año de servicio de 2000, La torre del vigía, México.

—, 2002, Anuario 2002 de los testigos de Jehová. Con el informe del año de servicio de 2001, La torre del vigía, México.

—, 2003, Anuario 2003 de los testigos de Jehová. Con el informe del año de servicio de 2002, La torre del vigía; México.

Higuera Bonfil, Antonio, 1997, A Dios las dendasy al alcalde las jaranas. Religión y politica en el Caribe mexicano, México, Universidad de Quintana Roo - CONACyT

—, 2001, "Uso de medios y expansión religiosa. Los testigos de Jehová", en Anuario 1 de la División de Estudios Internacionales y Humanidades, Universidad de Quintana Roo, México.

La torre del vigía, 2003, Programa de la asamblea internacional de los Testigos de Jehová 2003-2004 “Demos gloria a Dios”, México.

Mann, Brenda M., 1972, “The great crowd: ethnography of Jehovah's witnesses", in Spradley, James P. and McCurdy Davis W. The cultural experience. Ethnography in complex society, Science Research Associates Inc., USA.

Watch Tower Bible and Tract Society of Pennsylvania, 2004, Anuario 2004 de los testigos de Jehová. Con el informe del año de servicio de 2003, La torre del vigía, México.

Watch Tower Bible and Tract Society of Pennsylvania, Watch Tower Bible and Tract Society of New York, International Bible Students Association, Libro de textos bíblicos para 1974. Con el informe el año de servicio 1973, también textos diarios y comentarios, Watch Tower Bible and Tract Society of Pennsylvania y Watch Tower Bible and Tract Society of New York, Estados Unidos de América.

International Bible Students Association, 1975, Libro de textos bíblicos para 1975. Con el informe el año de servicio 1974, también textos diariosy comentarios, Watch Tower Bible and Tract Society of Pennsylvania, Watch Tower Bible and Tract Society of New York, Estados Unidos de América.

International Bible Students Association,1976, Libro de textos bíblicos para 1976. Con el informe el año de servicio 1975, también textos diarios y comentarios, Watch Tower Bible and Tract Society of Pennsylvania,

Watch Tower Bible and Tract Society of New York, Estados Unidos de América. 
International Bible Students Association, 1977, Libro de textos bíblicos para 1977. Con el informe el año de servicio 1976, también textos diarios y comentarios, Watch Tower Bible and Tract Society of Pennsylvania,Watch Tower Bible and Tract Society of New York, Estados Unidos de América.

International Bible Students Association, 1978, Libro de textos bíblicos para 1978. Con el informe el año de servicio 1977, también textos diarios y comentarios, Watch Tower Bible and Tract Society of Pennsylvania, Watch Tower Bible and Tract Society of New York, Estados Unidos de América.

International Bible Students Association, 1979, Libro de textos bíblicos para 1979. Con el informe el año de servicio 1978, también textos diarios y comentarios, Watch Tower Bible and Tract Society of Pennsylvania,Watch Tower Bible and Tract Society of New York, Estados Unidos de América.

International Bible Students Association, 1980, Libro de textos bíblicos para 1980. Con el informe el año de servicio 1979, también textos diarios y comentarios, Watch Tower Bible and Tract Society of Pennsylvania, Watch Tower Bible and Tract Society of New York, Estados Unidos de América.

International Bible Students Association, 1981, Libro de textos bíblicos para 1981. Con el informe el año de servicio 1980, también textos diarios y comentarios, Watch Tower Bible and Tract Society of Pennsylvania, Watch Tower Bible and Tract Society of New York, Estados Unidos de América.

International Bible Students Association, 1982, Libro de textos bíblicos para 1982. Con el informe el año de servicio 1981, también textos diarios y comentarios, Watch Tower Bible and Tract Society of Pennsylvania, Watch Tower Bible and Tract Society of New York, Estados Unidos de América.

International Bible Students Association, 1983, Libro de textos bíblicos para 1983. Con el informe el año de servicio 1982, también textos diarios y comentarios, Watch Tower Bible and Tract Society of Pennsylvania, Watch Tower Bible and Tract Society of New York, Estados Unidos de América.

International Bible Students Association, 1984, Libro de textos bíblicos para 1984. Con el informe el año de servicio 1983, también textos diarios y comentarios, Watch Tower Bible and Tract Society of Pennsylvania, Watch Tower Bible and Tract Society of New York, Estados Unidos de América.

International Bible Students Association, 1985, Libro de textos bíblicos para 1985. Con el informe el año de servicio 1984, también textos diarios y comentarios, Watch Tower Bible and Tract Society of Pennsylvania, Watch Tower Bible and Tract Society of New York, Estados Unidos de América.

International Bible Students Association, 1986, Libro de actividades 1986. Con el informe del año de servicio 1985, Watch Tower Bible and Tract Society of Pennsylvania, Watch Tower Bible and Tract Society of New York, Estados Unidos de América.

International Bible Students Association, 1987, Anuario de los testigos de Jehová para 1987. Con el informe del año de servicio de 1986, Watch Tower Bible and Tract Society of Pennsylvania, Watch Tower Bible and Tract Society of New York, Estados Unidos de América.

International Bible Students Association, 1988, Anuario de los testigos de Jehová para 1988. Con el informe del año de servicio de 1987, Watch Tower Bible and Tract Society of Pennsylvania, Watch Tower Bible and Tract Society of New York, Estados Unidos de América.

International Bible Students Association, 1989, Anuario de los testigos de Jehová para 1989. Con el informe del año de servicio de 1988, Estados Unidos de América.

Wilson, Bryan, 1970, Sociología de las sectas religiosas, Ed. Guadarrama, Biblioteca para el Hombre Actual, Madrid. 